\title{
Pneumocystis jiroveci and cytomegalovirus co-infection in an immunocompromised patient
}

\author{
D Simon, ${ }^{1} \mathrm{MB} \mathrm{ChB}, \mathrm{FCP}, \mathrm{MMed} ; \mathbf{K}$ E Greyling, ${ }^{2} \mathrm{MB} \mathrm{ChB}, \mathrm{FCP}, \mathrm{MMed}$, Cert Infectious Diseases; \\ E M Irusen, ${ }^{1} \mathrm{MB}$ ChB, FCP, PhD; J Rigby, ${ }^{3} \mathrm{MB}$ ChB, FC Path (SA) Anat; J J Taljaard, ${ }^{2} \mathrm{MB}$ ChB, MMed, DTM\&H; \\ C F N Koegelenberg, ${ }^{1} \mathrm{MB}$ ChB, FCP, FRCP, PhD

\begin{abstract}
${ }^{1}$ Division of Pulmonology, Department of Medicine, Stellenbosch University and Tygerberg Academic Hospital, Cape Town, South Africa
${ }^{2}$ Division of Infectious Diseases, Department of Medicine, Stellenbosch University and Tygerberg Academic Hospital, Cape Town, South Africa

${ }^{3}$ Division of Anatomical Pathology, Department of Pathology and National Health Laboratory Service, Stellenbosch University and Tygerberg Academic Hospital,
\end{abstract} \\ Cape Town, South Africa
}

Corresponding author: D Simon (siya_bonga@hotmail.com)

\begin{abstract}
Pneumocystis jiroveci pneumonia (PCP) and cytomegalovirus (CMV) are opportunistic infection seen in patients with advanced immunocompromised states, such as HIV infection. We present a case of PCP-CMV co-infection in a patient with newly diagnosed HIV disease. The presence of $\mathrm{CMV}$ in the context of another opportunistic respiratory tract infection is often presumed non-invasive and not treated. Our report highlights that this is not always the case. Invasive CMV disease can be easily misdiagnosed and remains a potentially fatal affliction. We postulate that the use of high-dose corticosteroids used as an adjunct in the treatment of serious PCP can lead to the reactivation of CMV infection and clinical disease. Moreover, we suggest that there may be a role for serial viral load measurement, analogous to protocols often utilised in solid-organ transplant patients receiving immunosuppression.
\end{abstract}

S Afr Respir J 2016;22(1):20-21. DOI: 10.7196/SARJ.2016.v22i1.40

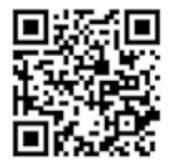

Pneumocystis jiroveci pneumonia (PCP) and cytomegalovirus (CMV) are opportunistic infections seen in patients with advanced immunocompromised states, including human immunodeficency virus (HIV). PCP and CMV pneumonitis present in a similar manner, but the diagnosis of CMV infection is often far more challenging. ${ }^{[1]}$ The poor sensitivity and specificity of CMV viral load often delays treatment of this potentially lethal co-infection. In addition, the presence of CMV in bronchoalveolar lavage (BAL) fluid or PCP polymerase chain reaction (PCR) in patients with pneumocystis pneumonia denotes a poor prognosis. ${ }^{[1]}$

\section{Case report}

A 48-year-old female presented with a 2-week history of a productive cough, dyspnoea and fever. She reported weight loss of $>10 \mathrm{~kg}$ over the preceding year. She was not known with any significant past medical history and had a 10 pack-year smoking history.

Clinical examination revealed an acutely ill patient who was tachypnoeic with oxygen saturation on room air of $84 \%$. Bilateral

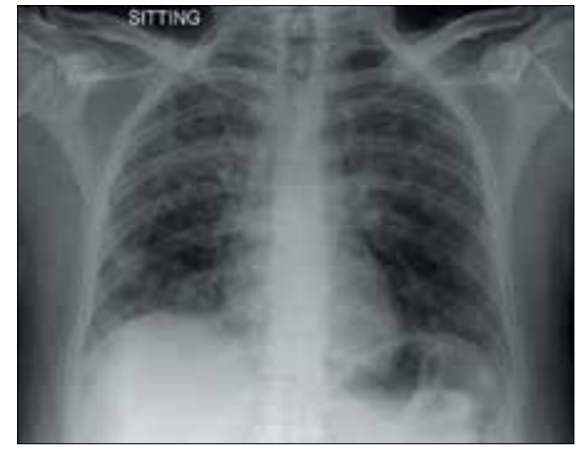

Fig. 1. Chest radiograph on admission. Note the diffuse reticulo-nodular infiltrates.

inspiratory crackles were present. The rest of her clinical examination was noncontributory.

A chest radiograph at presentation revealed bilateral interstitial infiltrates (Fig. 1). An HIV enzyme-linked immunosorbent assay test (ELISA) confirmed that she was HIV infected. Her serum lactate dehydrogenase was raised at $646 \mathrm{IU} / \mathrm{L}$ and her CD4 count was $87 \mathrm{cell} / \mathrm{mm}^{3}$ Sputum analysis confirmed $P$. jiroveci cysts. No other pathogens (including Mycobacterium tuberculosis) were identified in her sputum.

The patient was initiated on high-dose oral trimethoprim-sulphomethoxazole and

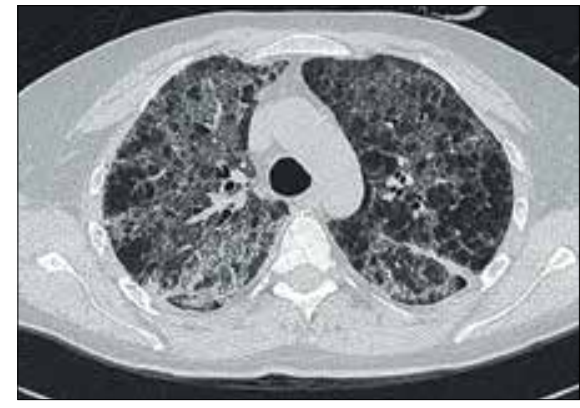

Fig. 2. A high-resolution computed tomography scan showing ground-glass infiltrates.

oral prednisone in addition to supplemental face-mask oxygen. She remained hypoxic and oxygen-dependent at the end of her 3-week treatment course. Her chest radiograph showed a worsening ground-glass pattern. A high-resolution computed tomography chest confirmed an interstitial process with a ground-glass appearance as well as honeycombing (Fig. 2).

The differential diagnosis at this point included cotrimoxazole-resistant $P$. jiroveci infection, other opportunistic infections and non-infective causes such as lymphocytic interstitial pneumonitis or nonspecific interstitial pneumonia. Her CMV viral load 


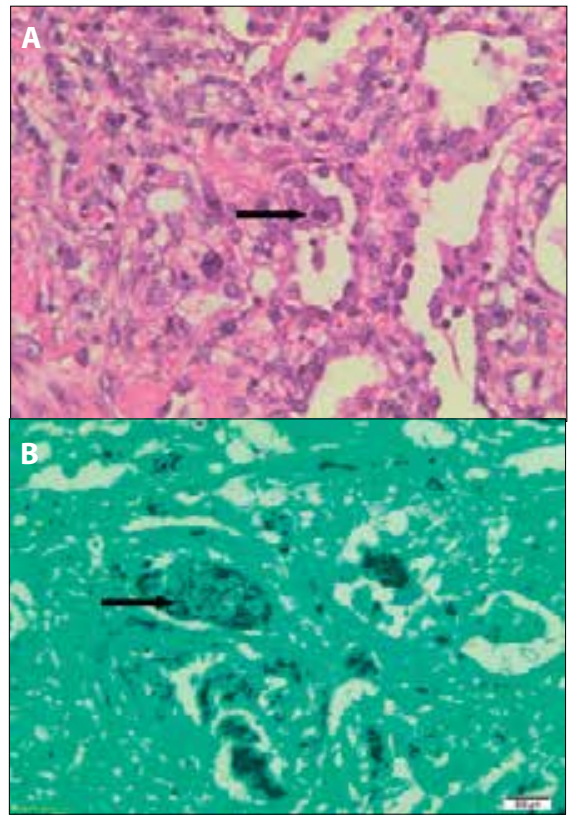

Fig. 3. The histology was diagnostic of CMV infection with (A) a diagnostic basophilic nuclear inclusion surrounded by a clear halo and indistinct basophilic cytoplasmic inclusions (HઐE). Immunohistochemistry confirmed diffuse nuclear and cytoplasmic positivity. (B) Black intra-alveolar cup-shaped cysts of P. jiroveci highlighted by Grocott's methenamine silver stain were also seen.

was 957768 copies $/ \mathrm{mL}$. She was deemed unfit for bronchoscopy due to persistent respiratory failure. An open lung biopsy demonstrated a frothy alveolar exudate with numerous cysts of $P$. jiroveci as well as CMV inclusion bodies (Fig. 3). Intravenous ganciclovir was initiated, but the patient died 4 days later despite full medical support, including mechanical ventilation.

\section{Discussion}

The advent of antiretroviral treatment has brought about a dramatic reduction in the number of HIV-infected patients presenting with opportunistic infections with P. jiroveci and CMV. Historically, the presence of CMV in the context of another opportunistic respiratory tract infection was presumed non-invasive and not treated. ${ }^{[2]}$ Evidence suggests no difference in outcome between CMV-treated and CMV-untreated groups. ${ }^{[2]}$
The majority of studies were, however, conducted prior to the use of corticosteroids as standard ancillary treatment for hypoxia in PCP infection. ${ }^{[3]}$ It is plausible that the use of high-dose corticosteroids can lead to the reactivation of CMV infection and clinical disease.

The diagnostic challenge is to distinguish between CMV infection and invasive CMV disease. CMV infection implies the presence of CMV evidenced by direct isolation, culture, seroconversion, a fourfold increase in titre, antigen detection or identification of CMV DNA by molecular techniques. In contrast, invasive CMV disease is characterised by an infectious syndrome and specific organ dysfunction together with pathological changes ${ }^{[4]}$ Radiological findings may include ground-glass attenuation, interstitial infiltrates (both reticular and/or nodular), dense consolidation, bronchial wall thickening and even apparent mass lesions. ${ }^{[1]}$

Toyoda et al. ${ }^{[3]}$ investigated the use of the total CMV viral burden in the serum as a marker for invasive CMV disease. Using quantitative PCR in a cohort of cardiac and renal transplant recipients, they found that there was a direct correlation between the CMV viral load and invasive disease. Diagnostic techniques have improved considerably since 1997 with real-time PCR currently the gold standard in determining viral load. Due to the absence of an internationally standardised reference reagent for quantitative PCR prior to 2010, it is impossible to compare studies using different assays from different laboratories in that era. Viral loads of $2000-5000 \mathrm{IU} / \mathrm{mL}$ in the context of a clinical syndrome consistent with CMV infection are generally believed to be consistent with invasive CMV disease in transplant patients. The Transplantation Society International CMV Consensus Group and the American Society of Transplantation guidelines on CMV infection in solid-organ transplantation recommend plasma blood viral load testing and state that laboratories must establish their own cut-offs to validate the trigger points used to initiate therapy ${ }^{[5]}$ We believe that a similar approach should be followed in other immunocompromised groups and that in-house cut-offs should be developed in conjunction with the local virology laboratory.

CMV viral load must be monitored using real-time quantitative PCR at baseline when steroids are initiated and then weekly thereafter until there is clinical improvement. An increase in viral load is often predictive of early disease and should be considered as an alternative to weekly testing in patients who are clinically deteriorating. ${ }^{[6]}$ All viral load testing should be followed by thorough clinical investigation e.g., fundoscopy, repeat chest radiography and if pneumonitis is suspected, a BAL for CMV quantitative PCR and appropriate cytological examination.

The index case not only demonstrates that true PCP and CMV pneumonitis can occur in a newly diagnosed HIV-infected patient, but also that CMV pneumonitis carries a high mortality despite appropriate treatment.

Consent: Informed consent to report this case was obtained from one of the patient's living relatives as the patient was on invasive ventilatory support and sedated at the time.

\section{References}

1. Salomon N, Gomez T, Perlman D, Laya L, Eber C, Mildvan D. Clinical features and outcome of HIV-related cytomegalovirus pneumonia. AIDS 1997;11(3):319-324

2. Jacobson MA, Mills J, Rush J, et al. Morbidity and mortality of patients with AIDS and first-episode Pneumocystis carinii pneumonia unaffected by concomitant pulmonary cytomegalovirus infection. Am Rev Respir Dis 1991;144(1):6-9.[http://dx.doi org/10.1164/ajrccm/144.1.6]

3. Toyoda M, Carlos JB, Galera OA, et al. Correlation of cytomegalovirus DNA levels with response to antiviral therapy in cardiac and renal allograft recipients. Transplantation 1997;63(7):957-963.

4. Kraft CS, Armstrong WS, Caliendo AM. Interpreting quantitative cytomegalovirus DNA testing: Understanding thelaboratory perspective. Clin Infect Dis 2012;54(12):17931797. [http://dx.doi.org/10.1093/cid/cis212]

5. Kotton CN, Kumar D, Caliendo AM, et al. Updated international consensus guidelines on the management of cytomegalovirus in solid-organ transplantation. Transplantation 2013;96(4):333-360. [http://dx.doi. org/10.1097/TP.0b013e31829df29d]

6. Emery VC, Sabin CA, Cope AV, et al. Application of viral-load kinetics to identify patients who develop cytomegalovirus disease after transplantation. Lancet 2000;355(9220):2032-2036. [http://dx.doi. org/10.1016/S0140-6736(00)02350-3] 